\title{
Provisionamento de Recursos de Hardware em Redes Híbridas de Acesso por Rádio com Base no Efeito de Maré
}

\author{
Igor W. S. Falcão ${ }^{1}$, Rafael F. Vieira ${ }^{1}$, Marcos C. da R. Seruffo ${ }^{1}$, Diego L. Cardoso ${ }^{1}$ \\ ${ }^{1}$ Instituto de Tecnologia - Universidade Federal do Pará (UFPA) \\ 66.075.110 - Belém - PA - Brasil \\ ${ }^{2}$ Departamento de Sistemas e Computação \\ Universidade Federal do Pará (UFPA) - Belém, PA - Brazil \\ \{igorufpa2013.4, fogarollirafael\}@gmail.com, \\ \{seruffo, diego\}@ufpa.br
}

\begin{abstract}
The C-RAN (Centralized Radio Access Network) architecture is a proposal to meet the high demand of fifth generation (5G) networks. Because the communication traffic has a dynamic behavior due to the "Tidal Effect"(phenomenon responsible for variation in network flow), the current architecture, D-RAN (Decentralized Radio Access Network) does not guarantee the efficient delivery of resources. This work presents a heuristic for the dimensioning of hardware resources considering the Number of Users Served and Aggregate Flow. The proposal addressed traffic variability, maximizing the efficiency of BBU (BaseBand Unit) and quantifying SCs (Small Cells) needed to cover the network demand.
\end{abstract}

Resumo. A arquitetura C-RAN (Centralized Radio Access Network) é uma proposta para atender à alta demanda das redes de quinta geração (5G). Devido o tráfego de comunicação possuir um comportamento dinâmico em decorrência do "Efeito de Maré" (fenômeno responsável pela movimentação do fluxo de rede), a arquitetura atual, o D-RAN (Decentralized Radio Access Network) não garante o fornecimento eficiente de recursos. Este trabalho apresenta uma heurística para o dimensionamento de recursos de hardware considerando o Número de Usuários Atendidos e Vazão Agregada. A abordagem atendeu a variabilidade do tráfego, maximizando a eficiência da BBU (BaseBand Unit) $e$ quantificando as SC's (Small Cells) necessárias para cobrir a demanda da rede.

\section{Introdução}

Com a expansão do LTE (Long Term Evolution) e os avanços no sistema de quinta geração $(5 \mathrm{G})$, a demanda por comunicação sem fio tem sido a força motriz por trás do progresso da tecnologia sem fio. Sob estas circunstâncias, o aumento no fluxo de dados previsto para os próximos anos [Khan et al. 2018] [Oughton et al. 2019] torna as redes de acesso por rádio descentralizadas (D-RAN) muito caras para as operadoras de serviços móveis [Khorsandi and Raffaelli 2018] e ineficientes quando se tem demanda de tráfego dinâmica. Esse comportamento do tráfego, denominado Efeito de Maré, gera o deslocamento do fluxo de comunicação em diferentes topologias de rede, especialmente em regiões que possuem migração diária da população [Yan et al. 2018] [Zhong et al. 2016]. 
A arquitetura de rede de acesso por rádio centralizada (C-RAN) é uma importante melhoria para a arquitetura de rede atual, bem como uma solução para o alto tráfego de dados previsto no 5G. O C-RAN, foi considerado por várias operadoras [Mobile 2011] e provedores de serviços nos últimos anos [Bian and Rao 2014], indicando um quadro economicamente favorável para implantação ultra compacta de SC's e de BBU's. Essa arquitetura centralizada também melhora a flexibilidade e permite uma operação de rede móvel mais robusta.

Apesar disso, a arquitetura apresenta sérios desafios (mapeamento e alocação de usuários, provisionamento de recursos de hardware, mapeamento de RRhs (Remote Radio Head) e implantação de BBU's [Khan et al. 2015] [Musumeci et al. 2016] que podem ser agravados com a não estacionariedade do usuário, conforme visto no Efeito de Maré. Nesse caso, a arquitetura deve ser dinâmica, o que significa que a capacidade da rede deve seguir o usuário e o dimensionamento da infraestrutura deve atender a necessidade de fluxo gerada pelos assinantes.

Embora a comunicação entre BBU e RRH não seja direta em termos físicos, pois na arquitetura C-RAN há um backhaul intermediando a comunicação entre núcleo e borda, existe uma conexão lógica um-para-um entre BBUs e RRHs em que uma BBU é designada para gerar/receber um sinal (por exemplo, quadro LTE ou WiMAX) para uma $\mathrm{RRH}$, onde esse mapeamento possa mudar com o tempo.

O estabelecimento da comunicação lógica entre as BBUs e RRHs de acordo com as condições fornecidas pelo Efeito de Maré, especialmente em redes híbridas, reforça que os estudos relacionados à flutuação do tráfego, sejam intensificados. Para tanto, uma Base de Dados com informações sobre a localização de usuários na cidade de New York foi utilizada, especificando o posicionamento exato dos usuários e auxiliando na obtenção de dados sobre o comportamento do tráfego na rede.

Neste trabalho foi desenvolvida uma heurística de seleção e determinação quantitativa de antenas, que devem ser conectadas para atender requisitos específicos de capacidade do cenário. A heurística se baseia em duas abordagens práticas, a primeira na Taxa de Transferência Agregada, enquanto a segunda na Quantidade de Usuários Conectados. A partir dessa metodologia, pretende-se especificar todo o processo de distribuição dos usuários na região de New York e quantificar o número de SC's necessárias para cobrir determinada capacidade do canal.

O restante do trabalho é estruturado da seguinte forma: A seção II apresenta os trabalhos correlatos a essa proposta. A seção III retrata a metodologia de desenvolvimento, apresentando: metadados, arquitetura proposta e os parâmetros de modelagem. Os resultados são mostrados na seção IV. Por fim, a seção V apresenta a conclusão.

\section{Trabalhos Correlatos}

As operadoras de rede estão se preparando para a migração da arquitetura descentralizada (D-RAN) para a centralizada (C-RAN). A migração implantará gradualmente o C-RAN, tornando esta um processo transparente ao usuário. A mudança no paradigma de rede não ocorrerá de forma abrupta, garantindo que os usuários operem sob ambas as arquiteturas.

A mudança de paradigma vem sendo bastante abordada pela literatura, ao passo de que sua abordagem requer uma análise mais aprofundada. Estudos sus- 
tentam que o C-RAN reduz significativamente os custos de implantação da RAN [Scholz and Grob-Lipski 2016], além de reduzir a sobrecarga do sistema e a ociosidade de recursos [Chang et al. 2016]. Por outro lado, há estudos que promovem o D-RAN porque ele opera sobre um backhaul realista, mas adverte contra a perda dos benefícios de centralização (redução de custos e facilidade de implantação de recursos de RAN) [Jaber et al. 2016].

A adoção de novas tecnologias juntamente com a expansão do C-RAN mostrase como um processo gradual. Há limitações de hardware que devem ser consideradas no cenário de 5G, particularmente tratando-se de: processamento [Zhang et al. 2016], latência [Yoshioka et al. 2016], capacidade [Idachaba 2016] e portas disponíveis para comunicação. A ideia principal é dedicar a implantação de arquiteturas centralizadas para as regiões altamente densificadas, que possuem um grande volume de tráfego, aumentando a capacidade e consequentemente, a QoS (Quality of Service).

Regiões densificadas são áreas com alta movimentação de usuários, que variam de acordo com o horário do dia [Pompili et al. 2015]. Esse comportamento é causado especialmente pela migração diária da população em regiões densificadas, provocando a flutuação espaço-temporal do tráfego na rede. Os padrões do efeito de maré podem ser facilmente encontrados em diferentes tipos de áreas, por exemplo, nas demandas de tráfego de entretenimento de alta largura de banda, como vídeo de alta definição e jogos online [Yan et al. 2018].

O posicionamento do usuário e a demanda por capacidade computacional são aspectos em constante intercorrência. No D-RAN tradicional, os recursos espectrais e de processamento de cada BS (Base Station) são usados apenas pelos usuários ativos em sua célula, causando sistemas ociosos em algumas áreas. Não há tamanho fixo de célula que otimize a cobertura geral de uma rede. Isto significa que o uso de SC's com o CRAN é bastante eficiente em termos de energia, bem como na utilização de recursos de processamento para demandas uniformemente distribuídas [Hajisami et al. 2015].

$\mathrm{Na}$ arquitetura C-RAN é possível implementar o provisionamento de recursos com reconhecimento de demanda, no qual os recursos da BBU são dinamicamente alocados para atender a carga móvel na rede. No quesito alocação de recursos de hardware a nível de RRH-BBU em 5G, diversos trabalhos na literatura apresentam soluções com foco em QoS, KPI (Key Performance Indicator) e número de PRB's (Physical Resource Block) [Fakhri et al. 2018] [Wang et al. 2017].

Já em [Yoshioka et al. 2016], um algoritmo de mapeamento dinâmico RRH-BBU na arquitetura C-RAN foi desenvolvido, simulando um cenário para verificar o desempenho do esquema de alocação conjunta de recursos. Os autores de [Pan et al. 2017] buscam resolver um problema de mapeamento de RRH e otimização, visando minimizar o consumo de energia da rede em um C-RAN centrado no usuário baseado em MIMO (Multiple-input and multiple-output). Em [Namba et al. 2012] os autores propuseram dois esquemas de troca de BBU-RRH para o C-RAN, utilizando algoritmo semi-estático para efetivar a combinação adaptativa e atender as demandas de tráfego de forma flexível.

Conforme referenciado, diversos trabalhos concentram seus esforços no balanceamento de carga em arquiteturas centralizadas, nas discussões sobre arquiteturas descentralizadas e na flutuação do fluxo de dados forma superficial. Contudo, a limitação 
de recursos em arquiteturas híbridas, o desligamento de RRHs dependendo da variação do tráfego (visando o melhor aproveitamento dos recursos de rede) e o provisionamento dinâmico de recursos são lacunas ainda pouco exploradas pela literatura científica, especialmente em se tratando de flexibilidade do tráfego de rede.

Os desafios acerca de alocação de recursos de hardware, posicionamento do tráfego, ociosidade de recursos e os problemas gerados pela mobilidade dos usuários podem ser efetivamente mitigados com propostas de centralização em redes híbridas. Para tanto, este trabalho tem como diferencial, o provisionamento dinâmico de recursos de hardware com a carga da rede flutuando em decorrência do efeito de maré. A ideia é estabelecer um dimensionamento eficiente de rede para suportar toda demanda recebida, sobretudo, solucionar as questões de limitação de recursos e intercorrência da arquitetura atual.

\section{Metodologia Desenvolvida}

O comportamento demográfico ao longo do tempo é um fator crucial para efetuar o dimensionamento de carga e o provisionamento de recursos. Em vista disso, uma fonte de dados baseada em ferramentas de redes sociais baseadas em localização (Location based social networks) [Kylasa et al. 2016], foi utilizada para armazenar informações de check-ins de usuários em diferentes localidades de New York (Queens, Manhattan, Bronx, Brooklyn e Staten Island). A coleta foi realizada no período de Abril de 2012 a Fevereiro de 2013, obtendo uma amostra de 227.428 posições (Longitude e Latitude).

O estudo sobre metadados forneceu uma visão mais realística da movimentação dos usuários. Para tanto, o quantitativo de usuários em cada distrito de New York foi normalizado e utilizado como parâmetro de entrada para modelagem. O posicionamento de usuários foi analisado em três momentos do dia (manhã, tarde e noite) e dessa forma, foi possível obter informações de densidade específicas da cada região conforme ilustrado na Tabela 1:

Tabela 1. Densidade de Usuários por período do dia.

\begin{tabular}{|c|c|c|c|c|}
\hline \multirow{2}{*}{ Distrito } & \multirow{2}{*}{$\begin{array}{c}\text { Área de NY } \\
\text { (781.1 km }\end{array}$} & \multicolumn{3}{|c|}{ Densidade Demografica $\left(\mathbf{P} / \mathbf{k m}^{2}\right)$} \\
\cline { 3 - 5 } & & Manhã & Tarde & Noite \\
\hline Queens & 280 & 34.8 & 29.4 & 30.9 \\
\hline Manhattan & 59.1 & 573.6 & 633.2 & 675.8 \\
\hline Bronx & 110 & 31.1 & 27.4 & 32 \\
\hline Brooklyn & 180 & 71.5 & 55.9 & 60.5 \\
\hline Staten Island & 152 & 5.2 & 6.5 & 6.3 \\
\hline
\end{tabular}

A Tabela 1 mostra a densidade populacional na cidade de New York em 3 períodos do dia. Essa relação utiliza o número de check-ins de usuários (identificando seu posicionamento) dividido pela área total de cada distrito. O objetivo é identificar as regiões mais densas e com isso, modelar o provisionamento de recursos com base nas demandas de rede. Via de regra, a demanda por recursos nas regiões mais densas são elevadas, o que significa que mais antenas devem estar ativas nessa região, enquanto nas regiões menos densas, para evitar a ociosidade de recursos, menos antenas devem ser ativadas. 


\subsection{Arquitetura}

Com base no cenário de New York, uma arquitetura híbrida é proposta agregando os principais componentes do C-RAN. A nível de núcleo, a BBU pool está localizada concentrando todas as unidades de processamento do modelo, que por sua vez, aproveita a enorme capacidade de transporte de sinal da fibra para o fronthaul que está sendo representado pelo Switch Óptico conforme visto a seguir.

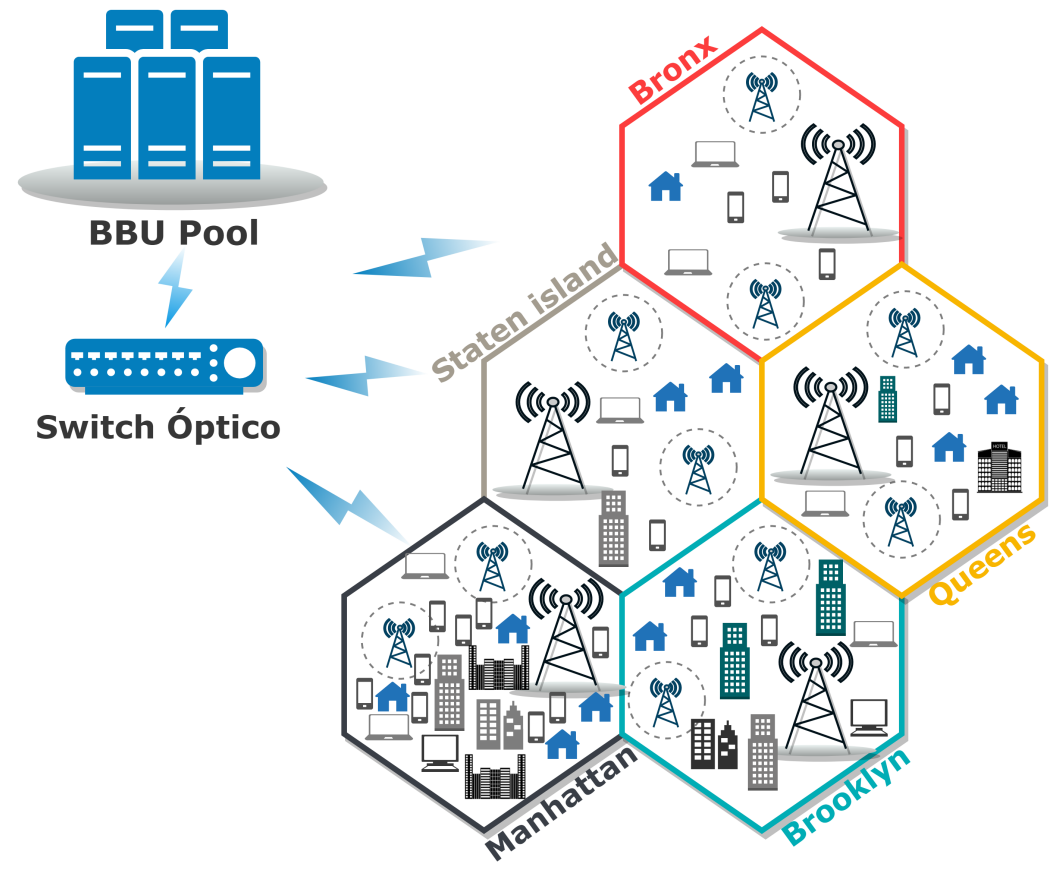

Figura 1. Arquitetura Proposta.

A arquitetura ilustrada na Figura 1 descreve o processo de comunicação proposto neste trabalho. A ideia é provisionar recursos para todos os centros urbanos da cidade de New York e,com base no fluxo de dados, alocar dinamicamente recursos de hardware para essa região. Cada setor possui características que impactam diretamente na quantidade de usuários, como áreas residenciais, áreas comerciais ou outras tipologias que constituem uma região densificada.

O distrito de Manhattan pode ser considerado uma área densificada, pois contém a maior concentração de usuários em relação aos demais distritos da New Yorkr em todos os períodos do dia, consequentemente um maior fluxo de dados. Já a região do Queens é tida como uma região menos densificada por apresentar uma baixa concentração de usuários. De maneira geral, cada célula possui um perfil de mobilidade de fluxo, cabendo ao modelo se adequar a esses fatores da arquitetura.

A flutuação do tráfego em diferentes períodos do dia, demanda um conjunto de RRHs correspondentes a macro BS para cobrir grandes áreas em extensões territoriais, bem como um conjunto de SC para abranger áreas reduzidas, maximizando a alocação dinâmica de recursos e reduzindo o descarregamento (offload) na BS.

\subsection{Parâmetros de Modelagem}

Conforme visto em [Sulyman et al. 2014], a perda de trajetória, em geral, é inversamente proporcional ao quadrado da frequência portadora. No planejamento celular, a perda 
de trajetória deve ser estimada para um ambiente de implantação e a cobertura celular é determinada com base nos ganhos da antena da estação base e da estação móvel, da energia irradiada isotrópica efetiva (EIRP), da largura de banda de RF, da modulação e de técnicas de codificação.

A perda de trajetória unidirecional em larga escala, em ambientes urbanos, pode ser estimada a partir do modelo Hata e sua extensão COST231 para frequência de portadora (FC) abaixo de $2 \mathrm{GHz}$ e do modelo Stanford University Interim (SUI) para FC acima de 2 GHz [Castro et al. 2010]. Logo, a relação sinal / interferência / ruído (SINR) de downlink sobre uma determinada subportadora $\mathrm{N}$ atribuída ao usuário K no SC ao qual está conectada, pode ser modelada da seguinte maneira:

$$
S I N R_{k}=\frac{P_{k, b(k)}}{\sigma^{2}+I_{k}}
$$

Em que $P k, b(k)$ é a potência recebida na subportadora $n$ atribuída ao usuário $\mathrm{k}$ por sua porção BS $\sigma^{2}$, é a potência de ruído térmico e $I_{k}$ é a interferência intercelular das SCs vizinhas. Assumiu-se que todos os SCs estão transmitindo com potência máxima $P$. A potência recebida no usuário $k$ de BS é $b(k)$ e pode ser calculada por meio da Equação 2, que relaciona a potência recebida de um nó com o resultado de transmissão, a potência e o desvanecimento do sinal. Este último cálculo é feito através do modelo de propagação SUI [Nassar et al. 2015]. Isso pode ser expresso como:

$$
P_{k, b(k)}=\frac{10^{\frac{\text { Pot }_{b(k)}-L_{S U I}}{10}}}{1000}
$$
equações:

O valor de $P k, b(k)$ é uma função dos três valores calculados pelas seguintes

$$
\begin{gathered}
L_{S U I}=A+10 \gamma \log \frac{d}{d_{o}}+S, d>d_{o}, \\
A=20 \log \frac{4 \pi d_{o}}{\lambda}, \\
\gamma=a-b h_{b}+\frac{C}{h_{b}}
\end{gathered}
$$

É considerado que cada usuário atinge a capacidade de Shannon, ou seja, a taxa de dados para o usuário $k$ é expressa em (6) como:

$$
C_{k}=B * \log 2\left(1+S I N R_{k}\right)
$$

Em que $B$ é a largura de banda do sistema. 


\section{Heurística Proposta}

A problemática desse trabalho pode ser divida em 2 passos. A primeira etapa cria todos os usuários $(u)$ e lista as possíveis RRHs disponíveis $\left(S_{t}\right)$, determinando o número de portas necessárias para cobrir uma determinada capacidade de fluxo agregado. Para isso, a primeira fase da heurística é descrita nos Algoritmos 1 e 2. Com base nessas informações, o Algoritmo 3 expressa: a implantação de RRH's, taxa de vazão agregada e o número de UE's cobertos pela Macro.

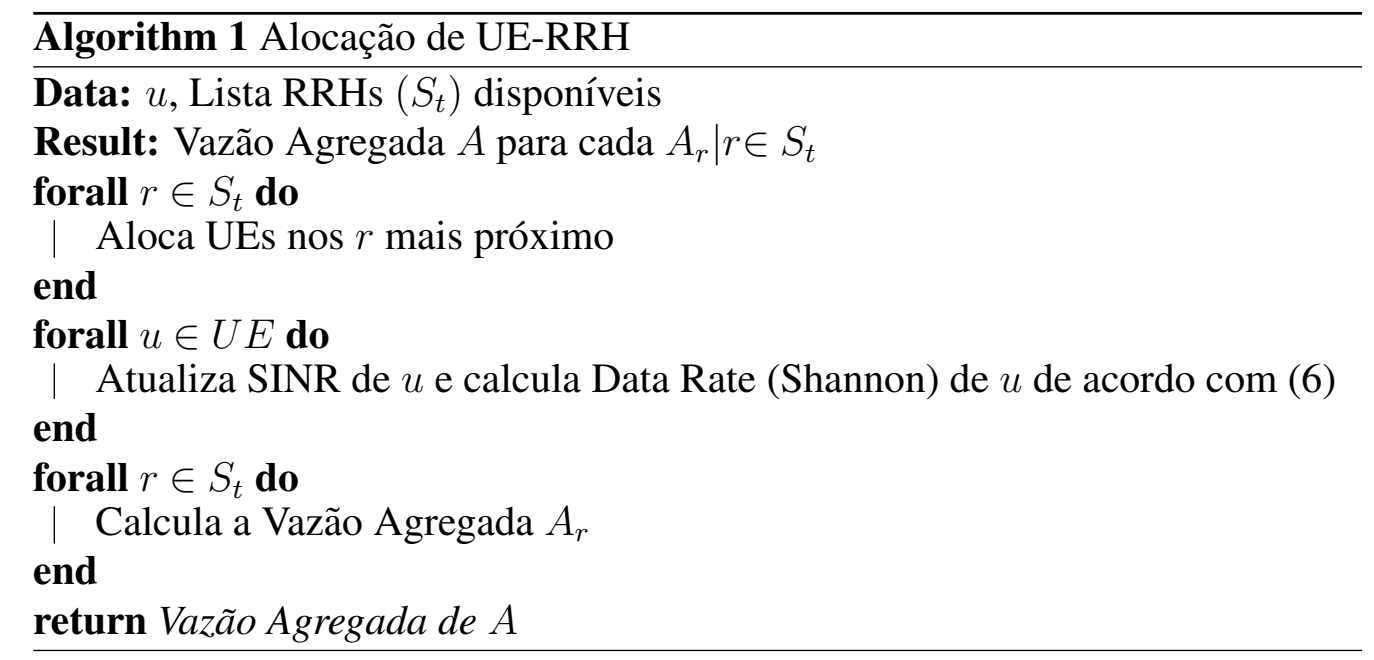

O algoritmo 1 descreve o método de alocação de UEs para os RRHs mais próximos, ou seja, as que estão disponíveis. Após esta fase, a capacidade máxima de cada UE é calculada (a interferência entre RRHs, e entre RRHs e UEs é calculada nesta fase) considerando os recursos disponíveis em cada RRH (PRBs), que são divididos igualmente. Ao final é gerada uma lista de RRHs com suas capacidades agregadas máximas, que servirão como entrada para o Algoritmo 2 descrito a seguir.

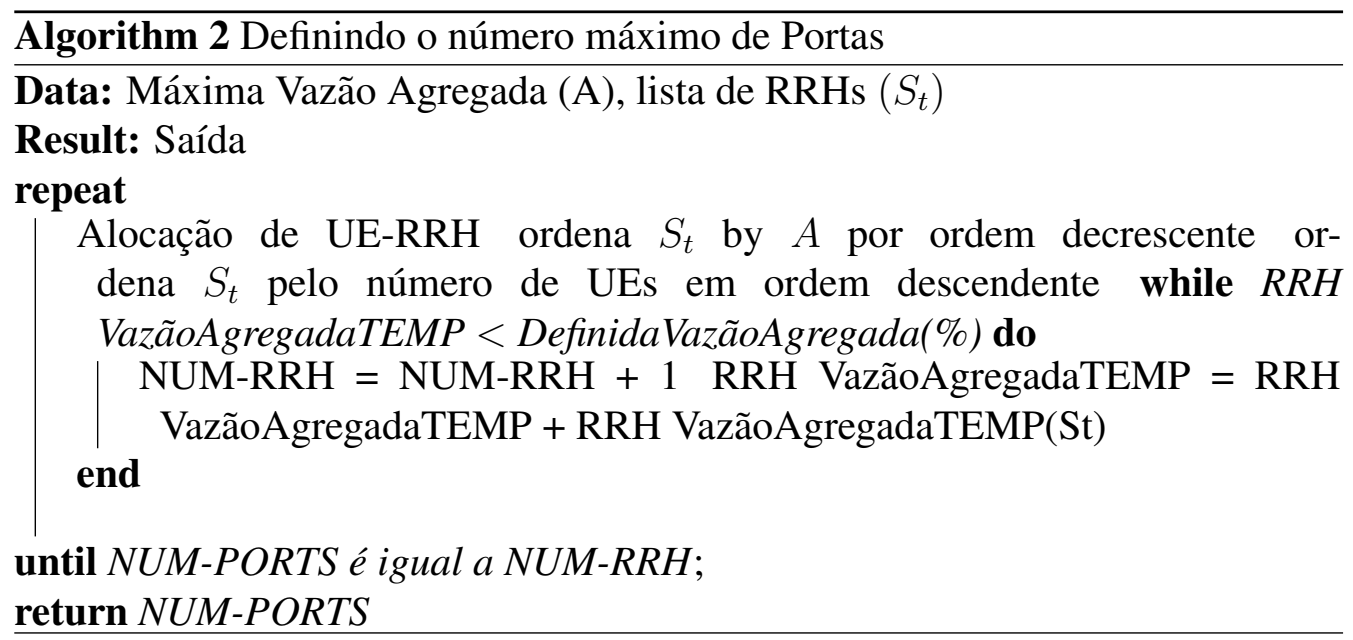

No Algoritmo 2, uma porcentagem máxima de taxa de transferência agregada é fornecida como entrada $(A)$, além da lista $\left(S_{t}\right)$ novamente. Nessa etapa, são consideradas diferentes posições de UEs e é contabilizado o número de RRHs necessários para cobrir 
determinada porcentagem da taxa de transferência agregada. Os testes são repetidos até que um número médio de portas seja obtido (equivalente ao número médio de RRHs necessários para descarregamento do fluxo).

Para determinar quais RRHs devem ser conectados, dois critérios foram usados: (i) a abordagem baseada vazão agregada, em que as RRHs com maior taxa de transferência agregada são priorizadas; (ii) a abordagem baseada na quantidade de usuário conectados, onde as RRHs com o maior número de usuários são priorizados. Ambas as abordagens devem respeitar os limites dos PRBs existentes nas RRHs disponíveis.

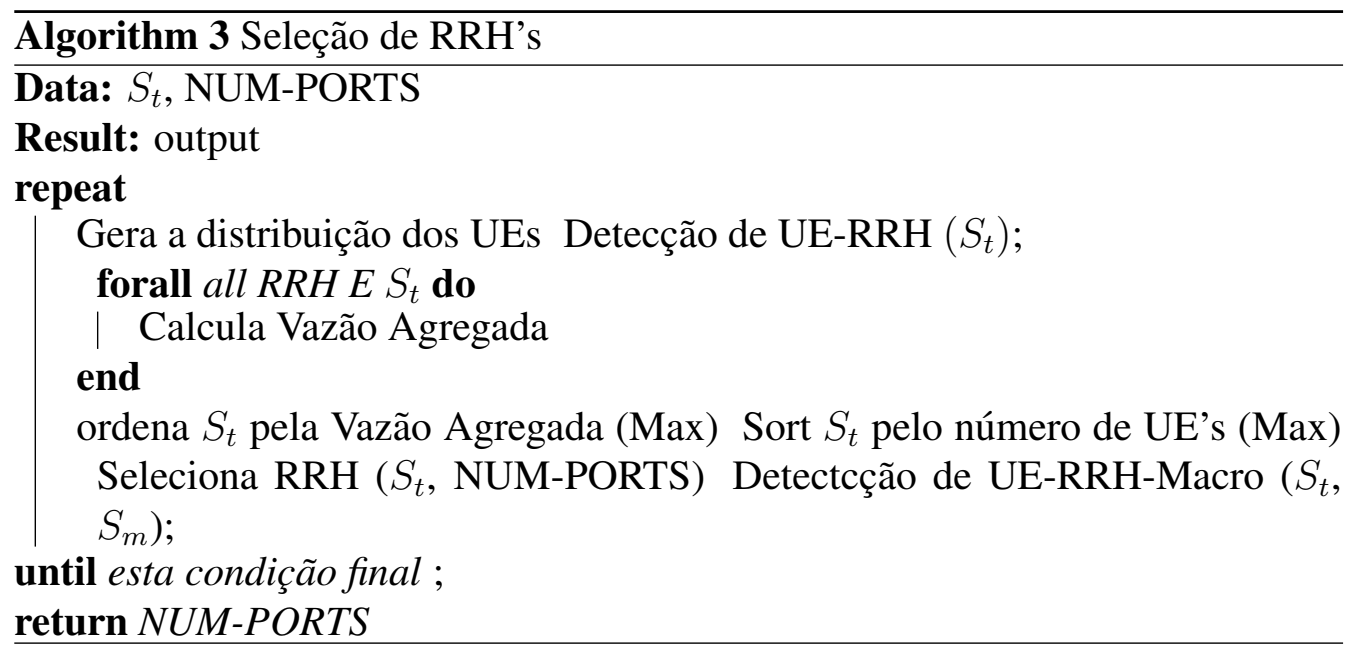

A segunda etapa é expressa pelo algoritmo 3, finalizando a execução da heurística empregada. Dito isso, com o número definido de usuários e o cenário como parâmetro de entrada, todos as RRHs são implantados e os UEs são alocados para essas RRHs. Com base no número de portas obtido com o algoritmo 2, as RRHs que atendem as duas abordagens (I, II) expressas anteriormente, as RRHs são eliminados e uma nova alocação de UEs e RRHs é feita. Ressalta-se que os UE's não atendido pelas RRHs (por motivos de posicionamento por exemplo), devem necessariamente ser cobertos pela Macro BS.

\section{Modelagem e Resultados}

O esquema de alocação de Usuários e o dimensionamento de recursos de hardware são

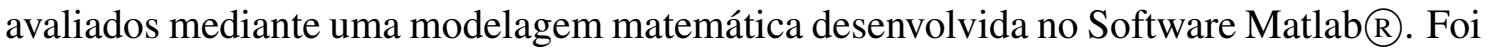
considerada uma rede móvel típica para avaliar o mapeamento de portas RRH-BBU, na qual, todo o tráfego de rede do cenário flutua durante períodos do dia.

O cenário proposto possui $4 \mathrm{~km}$ de extensão e é subdividido conforme a classificação por distritos da cidade de New York. São posicionadas aleatoriamente 100 small cells, uma macrocell (D-RAN) e 500 UE's com características homogêneas, o que significa que os UE's possuem os mesmo requisitos de QoS. As RRH's são posicionadas com base na distância Euclidiana. As dimensões do cenário foram normalizadas conforme os limites territoriais da região de New York. Os demais parâmetros da modelagem são listadas na Tabela 02:

Esta análise teve como base o movimento de usuários em três períodos do dia. O objetivo é analisar o comportamento da arquitetura proposta e verificar se há necessi- 
Tabela 2. Parâmetros de Modelagem.

\begin{tabular}{|l|l|}
\hline Parâmetros & Valor \\
\hline Largura de Banda $(B)$ & $180 \mathrm{kHz}$ \\
\hline Perda de Propagação (Macrocell) & COST231 \\
\hline Perda de Propagação (Smallcell) & SUI-TYPE A \\
\hline Potência de Transmissão (Macrocell) & $43 \mathrm{dBm}$ \\
\hline Potência de Transmissão (Smallcell) & $23 \mathrm{dBm}$ \\
\hline Área Total do Cenário & $4 \mathrm{~km}^{2}$ \\
\hline Altura da BS & $16 \mathrm{~m}$ \\
\hline Área de Cobertura (Small) & $150 \mathrm{~m}$ \\
\hline Área de Cobertura (Macro) & $4 \mathrm{~km}$ \\
\hline Intervalo de Confiança & $95 \%$ \\
\hline Número de experimentos & 30 \\
\hline
\end{tabular}

dade de maiores investimento na expansão da BBU de um determinado BBU Pool, para isso, é importante responder a pergunta: Quantas BSs são necessários suportar o tráfego dinâmico da rede considerando o efeito de maré?

O efeito de maré em regiões densificadas como New York, gera alta variabilidade do fluxo de rede com a movimentação dos usuários, provocando a flutuação do tráfego de rede. Tomando como base a heurística desenvolvida (baseada na Vazão agregada e no número de Usuários), foram considerados limiares para representar a capacidade total do cenário, fixados em: 20\%, 40\%, 60\%, 80\%, e 100\% da vazão agregada, conforme visto na Figura 02.

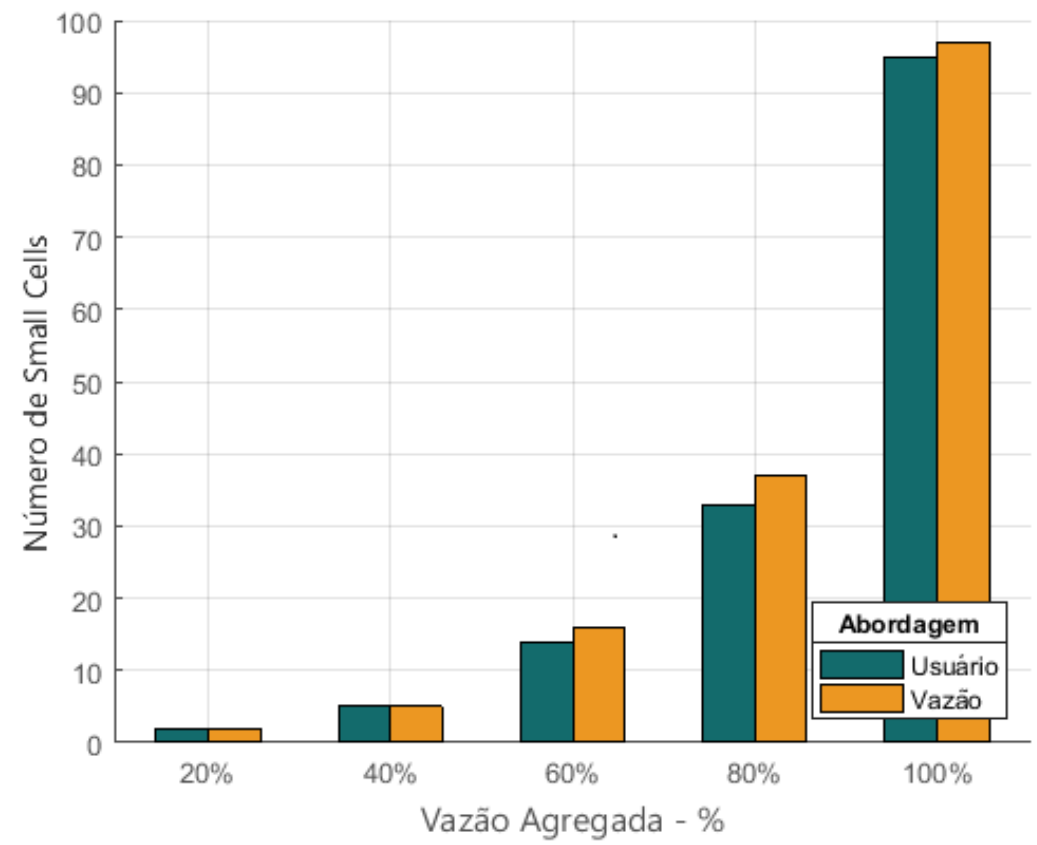

Figura 2. Número de Small Cells Selecionadas.

A eficiência do esquema de alocação é avaliada com base nas duas abordagens 
(Usuário e Vazão), entretanto, a abordagem de usuários, obteve um número menor de RRH's a partir de $60 \%$ da vazão agregada (Figura 02). Com o aumento da capacidade agregada, a abordagem de Vazão requer um número maior de RRHs selecionadas para atender a mesma demanda da rede.

Considerando o número médio de usuários por Small Cell, a abordagem de Usuários apresentou desempenho superior, embora o número de antenas selecionadas tenha sido menor. Esse resultado é comprovado pelo número médio de usuários por RRH que, independente da vazão agregada, é maior em $9.1 \%$ durante os períodos avaliados (manhã, tarde e noite). Este fato é esperado em uma rede densificada, onde é mais fácil cobrir mais usuários que estão na área de cobertura das mesmas RRH.

Em geral, a carga de tráfego para células diferentes flutua muito ao longo do tempo, quando a RRH está sob condições elevadas de tráfego (muitos usuários conectados ou alta taxa de dados), mais recursos são necessários, no entanto, há uma limitação de recursos na BBU. Apesar disso, com baixa carga os recursos podem ser dinamicamente realocadas (através do esquema de alocação adaptativa) para outras RRHs com maior carga de tráfego naquele momento e, com isso, o desbalanceamento de carga acarretado pelo efeito de maré, pode ser efetivamente resolvido.

\subsection{Abordagem baseada em Usuários (seleção de RRH)}

Esta abordagem apresenta o número médio de usuários conectados a todas as RRHs selecionadas, o que corresponde ao número de portas definidas com base na Vazão Agregada do Cenário. $\mathrm{O}$ aumento no número de usuários não segue a mesma tendência da vazão média, como pode ser visto na Figura 03. Nota-se que o comportamento do usuário variou conforme a Capacidade do Cenário. Esse aumento promoveu uma vazão média mais elevada no período da manhã, Figura 03 (b), o que significa que os UE's estão mais concentrados, logo, podem ser cobertos com maior vazão agregada.

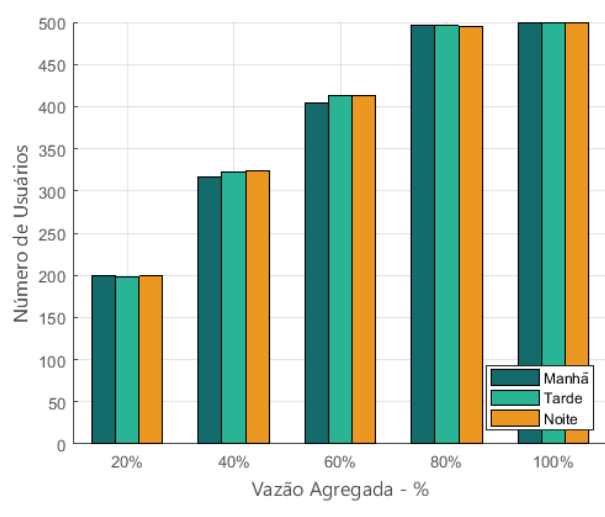

(a) Número médio de Usuários

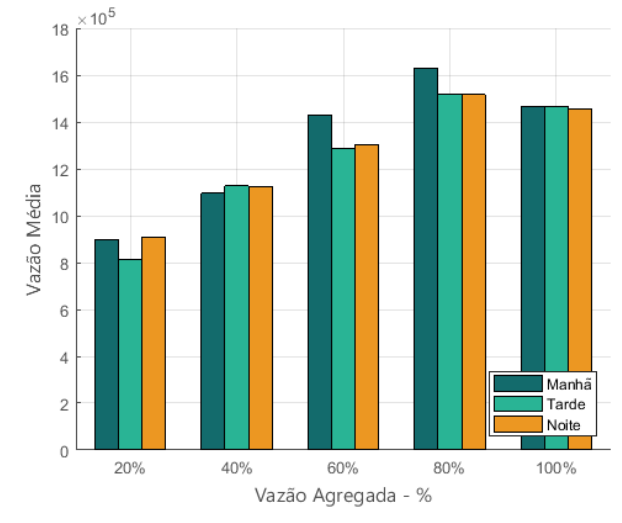

(b) Vazão Média

Figura 3. Desempenho para a abordagem baseada no Usuário

Esta abordagem seleciona as antenas nas áreas de maior concentração de usuários, característica de regiões densificadas. Observa-se que em 100\% da vazão agregada, todos os usuários foram atendidos, embora no período da manhã a vazão média tenha um comportamento mais instável. 
Vale ressaltar que os usuários localizados nas áreas de menor concentração se conectam nas antenas de menor capacidade, enquanto aqueles que não estão no raio de cobertura das RRHs, são encaminhados para a macro BS. No estado de menor densidade de usuários, o número de RRHs selecionadas será reduzido, o que garante uma distribuição eficaz de antenas e menor variabilidade da Vazão Média.

\subsection{Abordagem baseada em vazão (seleção de RRH)}

$\mathrm{Na}$ abordagem focada na Vazão, o número médio de usuários conectados a todas as RRHs selecionados, o que corresponde ao número de portas definidas com base e na porcentagem da vazão média, conforme visto na Figura 3 (a) e (b).

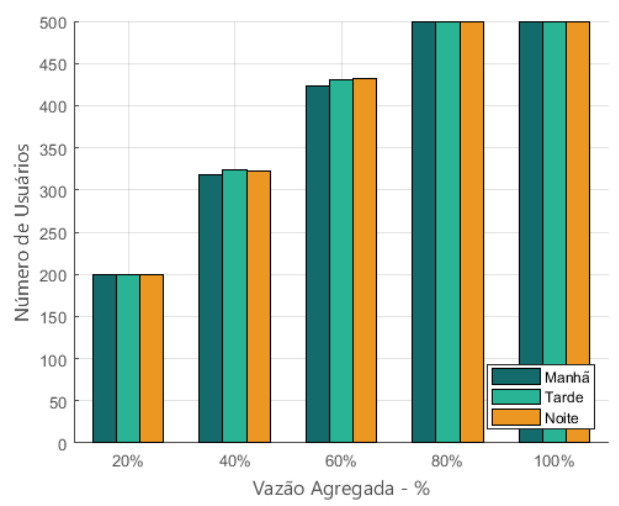

(a) Número médio de Usuários

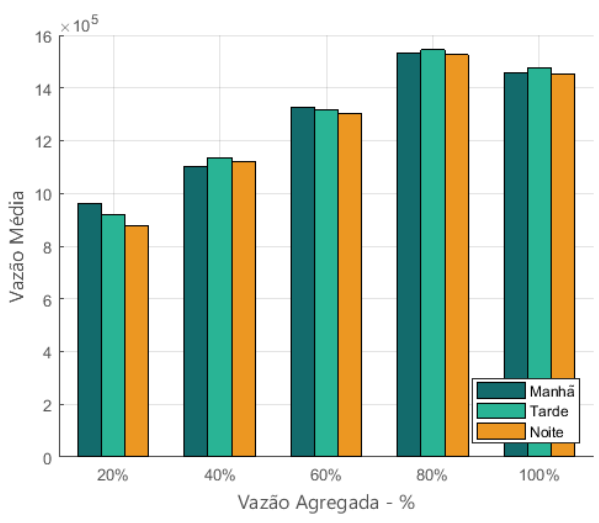

(b) Vazão Média

Figura 4. Desempenho para a abordagem baseada na Vazão

Nessa abordagem, a seleção de RRHs foi mais densa em todos os momentos do dia, proporcionando melhor cobertura de usuários a partir de $60 \%$ da vazão agregada. Assim, quanto maior o número de usuários conectados, maior a vazão média, exceto para $100 \%$ da vazão agregada como visto na Figura 04 (b). Para esta avaliação, há casos em que os usuários estão desconectados, todavia, os usuários que não se conectam às SCs são conectados à macro BS. Em relação à variabilidade da vazão média, as distâncias dos usuários para as RRHs, o raio de cobertura da antena e qualidade do sinal, são fatores determinantes para atender a capacidade do cenário.

\section{Conclusão}

A alocação e provisionamento de recursos de hardware é extremamente importante para o cenário de arquiteturas híbridas, que necessariamente devem cooperar para atender as necessidades dos usuários. Este trabalho analisou o comportamento do tráfego de rede na cidade de New York, constatando que a variabilidade do tráfego e o deslocamento do fluxo de rede são desafios enfrentados pela arquitetura de rede atual. Foram propostas duas abordagens que focam no dimensionamento de recursos da BBU, considerando o movimento de usuários ao longo do dia. Ambas tiveram desempenho satisfatórios para o cenário, conseguindo reduzir o número de Small Cells selecionadas e atendendo todos os usuários a partir de $80 \%$ da Capacidade do Cenário.

Os resultados obtidos mostram que é possível dimensionar o número de SCs que precisam ser ativadas ao mesmo tempo e, portanto, o número de portas necessárias para 
cobrir determinada capacidade do cenário, maximizando a multiplexação e eficiência no uso do processamento da BBU. Em trabalhos futuros podem ser consideradas outras abordagens para o esquema de provisionamento de recursos, levando em consideração Probabilidade de Bloqueio de Portas (visando a limitação de recursos da BBU) ou um uma heurística de Machine Learning (Aprendizado de Máquina) para efetuar as regras de dimensionamento conforme as distribuições dos usuários.

\section{Referências}

Bian, Y. Q. and Rao, D. (2014). Small cells big opportunities. Global Business Consulting. Huawei Technologies Co., Ltd.

Castro, B., Gomes, I., Ribeiro, F., and Cavalcante, G. (2010). Cost231-hata and sui models performance using a lms tuning algorithm on $5.8 \mathrm{ghz}$ in amazon region cities. In Proceedings of the Fourth European Conference on Antennas and Propagation, pages 1-3. IEEE.

Chang, W., Xie, T., Zhou, F., Tian, J., and Zhang, X. (2016). A prefiltering c-ran architecture with compressed link data rate in massive mimo. In 2016 IEEE 83rd Vehicular Technology Conference (VTC Spring), pages 1-6. IEEE.

Fakhri, Z. H., Khan, M., Sabir, F., and Al-Raweshidy, H. (2018). A resource allocation mechanism for cloud radio access network based on cell differentiation and integration concept. IEEE Transactions on Network Science and Engineering, 5(4):261-275.

Hajisami, A., Tran, T. X., and Pompili, D. (2015). Dynamic provisioning for high energy efficiency and resource utilization in cloud rans. In 2015 IEEE 12th International Conference on Mobile Ad Hoc and Sensor Systems, pages 471-472. IEEE.

Idachaba, F. E. (2016). 5g networks: Open network architecture and densification strategies for beyond 1000x network capacity increase. In 2016 Future Technologies Conference (FTC), pages 1265-1269. IEEE.

Jaber, M., Owens, D., Imran, M. A., Tafazolli, R., and Tukmanov, A. (2016). A joint backhaul and ran perspective on the benefits of centralised ran functions. In 2016 IEEE International Conference on Communications Workshops (ICC), pages 226-231. IEEE.

Khan, M., Alhumaima, R., and Al-Raweshidy, H. (2015). Quality of service aware dynamic bbu-rrh mapping in cloud radio access network. In 2015 International Conference on Emerging Technologies (ICET), pages 1-5. IEEE.

Khan, R., Al-Hadi, A. A., and Soh, P. J. (2018). Recent advancements in user effect mitigation for mobile terminal antennas: A review. IEEE Transactions on Electromagnetic Compatibility, (99):1-9.

Khorsandi, B. M. and Raffaelli, C. (2018). Bbu location algorithms for survivable 5g c-ran over wdm. Computer Networks, 144:53-63.

Kylasa, S. B., Kollias, G., and Grama, A. (2016). Social ties and checkin sites: connections and latent structures in location-based social networks. Social Network Analysis and Mining, 6(1):95.

Mobile, C. (2011). C-ran: the road towards green ran. White Paper, ver, 2:1-10. 
Musumeci, F., Bellanzon, C., Carapellese, N., Tornatore, M., Pattavina, A., and Gosselin, S. (2016). Optimal bbu placement for $5 \mathrm{~g}$ c-ran deployment over wdm aggregation networks. Journal of Lightwave Technology, 34(8):1963-1970.

Namba, S., Warabino, T., and Kaneko, S. (2012). Bbu-rrh switching schemes for centralized ran. In 7th International Conference on Communications and Networking in China, pages 762-766. IEEE.

Nassar, A. T., Sulyman, A. I., and Alsanie, A. (2015). Radio capacity estimation for millimeter wave $5 \mathrm{~g}$ cellular networks using narrow beamwidth antennas at the base stations. International Journal of Antennas and Propagation, 2015.

Oughton, E. J., Frias, Z., van der Gaast, S., and van der Berg, R. (2019). Assessing the capacity, coverage and cost of 5g infrastructure strategies: Analysis of the netherlands. Telematics and Informatics.

Pan, C., Zhu, H., Gomes, N. J., and Wang, J. (2017). Joint precoding and rrh selection for user-centric green mimo c-ran. IEEE Transactions on wireless Communications, 16(5):2891-2906.

Pompili, D., Hajisami, A., and Viswanathan, H. (2015). Dynamic provisioning and allocation in cloud radio access networks (c-rans). Ad Hoc Networks, 30:128-143.

Scholz, S. and Grob-Lipski, H. (2016). Reallocation strategies for user processing tasks in future cloud-ran architectures. In 2016 IEEE 27th Annual International Symposium on Personal, Indoor, and Mobile Radio Communications (PIMRC), pages 1-6. IEEE.

Sulyman, A. I., Nassar, A. T., Samimi, M. K., MacCartney, G. R., Rappaport, T. S., and Alsanie, A. (2014). Radio propagation path loss models for 5g cellular networks in the $28 \mathrm{ghz}$ and $38 \mathrm{ghz}$ millimeter-wave bands. IEEE Communications Magazine, 52(9):78-86.

Wang, K., Zhou, W., and Mao, S. (2017). On joint bbu/rrh resource allocation in heterogeneous cloud-rans. IEEE Internet of Things Journal, 4(3):749-759.

Yan, B., Zhao, Y., Yu, X., Wang, W., Wu, Y., Wang, Y., and Zhang, J. (2018). Tidaltraffic-aware routing and spectrum allocation in elastic optical networks. Journal of Optical Communications and Networking, 10(11):832-842.

Yoshioka, S., Inoue, Y., Suyama, S., Kishiyama, Y., Okumura, Y., Kepler, J., and Cudak, M. (2016). Field experimental evaluation of beamtracking and latency performance for $5 \mathrm{~g}$ mmwave radio access in outdoor mobile environment. In 2016 IEEE 27th Annual International Symposium on Personal, Indoor, and Mobile Radio Communications (PIMRC), pages 1-6. IEEE.

Zhang, J., Zhang, X., Yan, Z., Li, Y., Wang, W., and Zhang, Y. (2016). Social-aware cache information processing for 5g ultra-dense networks. In 2016 8th International Conference on Wireless Communications \& Signal Processing (WCSP), pages 1-5. IEEE.

Zhong, Z., Hua, N., Tornatore, M., Li, Y., Liu, H., Ma, C., Li, Y., Zheng, X., and Mukherjee, B. (2016). Energy efficiency and blocking reduction for tidal traffic via stateful grooming in ip-over-optical networks. Journal of Optical Communications and Networking, 8(3):175-189. 